\title{
The Role of Autoimmunity on the Relation Between Erosions and Bone Mineral Density in Rheumatoid Arthritis: A Clinical Research
}

Margaux Moret ( $\sim$ m.moret@chru-nancy.fr )

Nancy-Universite: Universite de Lorraine https://orcid.org/0000-0001-8448-525X

Caroline Morizot

Nancy-Universite: Universite de Lorraine

Marcelo de Carvalho Bittencourt

IMoPA: Ingenierie Moleculaire et Physiopathologie Articulaire

Edem Allado

Nancy-Universite: Universite de Lorraine

Isabelle Chary-Valckenaere

IMoPA: Ingenierie Moleculaire et Physiopathologie Articulaire

Damien Loeuille

IMoPA: Ingenierie Moleculaire et Physiopathologie Articulaire

\section{Research article}

Keywords: Rheumatoid arthritis, Erosion, Bone mineral density, Auto-immunity, ACPA

Posted Date: September 29th, 2021

DOI: https://doi.org/10.21203/rs.3.rs-934180/v1

License: @ (i) This work is licensed under a Creative Commons Attribution 4.0 International License. Read Full License 


\section{Abstract}

Introduction

Both erosions and osteoporosis are present in rheumatoid arthritis and are related to RANK-L pathway activation. The aim of the study was to evaluate the relationship between erosion and bone mineral density (BMD) in RA and whether it can be driven by autoimmunity.

Patients and methods

Patients followed in the Department of Rheumatology between January 2008 and May 2019 satisfied the 1987 ACR or 2010 ACR-EULAR criteria. Erosions were evaluated by the modified Sharp/van der Heidje erosion score (SHSe) on radiographs and bone mineral density (BMD) in $\mathrm{g} / \mathrm{cm}^{2}$ and by the T-score at the hip on DXA. The presence and titers of ACPA as well as rheumatoid factor (RF) and anti-nuclear antibodies (ANAs) were recorded at intervals of less than 2 years for both DXA and radiography.

Results

A total of 149 patients met the inclusion criteria. A total of $61.1 \%$ were ACPA positive, $79.9 \%$ were erosive and $10.7 \%$ had a hip T-score $\leq-2.5$. ACPA status but not titers was associated with a higher erosion score (63.0 (53.2) for ACPA + vs. 45.5 (44.1) for ACPA - $(p=0.04)$ ). ACPA titers were associated with lower BMD at the hip (value $-0.216 ; p=0.01)$ but not with T-score. A higher erosion score was associated with a lower BMD ( $R^{2}: 0,049$ and value: $\left.-0.222 ; p=0.009\right)$ and Tscore $\left(R^{2}: 0,158\right.$ and value $\left.-0.397 ; p<0.0001\right)$ at the hip. In linear regression, erosion and systemic bone loss were still associated with but not driven by ACPA status or titer. RF and ANA did not demonstrate any role in this association.

Conclusion

We showed that the relationship between erosion and bone mineral density associated with RA does not seem to be driven by ACPA or other autoimmunity parameters. However, the presence of ACPA or erosion should lead to osteoporosis assessment.

\section{Introduction}

Rheumatoid arthritis (RA) is the most frequent chronic inflammatory rheumatism, with a prevalence of $1 \%$ worldwide ${ }^{(1)}$

(2). It is characterized by the presence of local and general inflammation and peripheral articular destruction combining joint space narrowing and erosion. Erosions are secondary to osteoclastic activation, partly induced by proinflammatory cytokines (IL1b, IL6, IL8, TNF-alpha) expressed in synovitis ${ }^{(3)(4)}$. It is well known that erosions are correlated with the presence and titer of anti-citrullinated peptide antibodies (ACPAs) on radiographs but also on ultrasonography examinations (US) ${ }^{(5)(6)(7)(8)}$. The number and severity of erosions are greater in ACPA-positive RA than in ACPA-negative RA patients based on the modified Sharp/van der Heidje erosion score (SHSe) evaluation ${ }^{(6)(7)(8)}$.

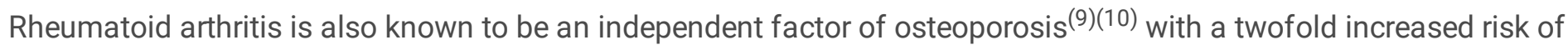
vertebral and nonvertebral fracture compared to the general population(11)(12)(13). Disease activity, disease duration, biological inflammation, smoking and corticosteroid intake are all risk factors contributing to bone loss and osteoporosis in RA patients ${ }^{(11)}$. Currently, the gold standard for the diagnosis and monitoring of osteoporosis is dualenergy X-ray absorptiometry (DXA) performed on the spine and hip. The prevalence of densitometric osteoporosis in RA varies from 10 to $50 \%$ according to the literature $\mathrm{e}^{(14)(15)(16)(17)(18)(19)}$. Additionally, it has already been demonstrated that ACPA titer or status are "significant predictors of bone mineral density (BMD) changes in the proximal femur"(20). 
Moreover, ACPA-positive status is associated with lower BMD at the spine and hip even in early RA ${ }^{(21)(22)}$. ACPAs are also associated with a higher likelihood of having a major fracture at 10-year or a hip fracture according to the FRAX (2) (23).

The physiopathology of erosion and bone loss in ACPA-positive patients is related to different pathways, such as direct activation of preosteoclast cells or immune cells (monocytes, macrophages, etc.) by ACPAs due to the presence of citrullinated peptides on their surface. As a consequence, TNF-alpha and receptor activator of nuclear factor kappa $B$ ligand (RANK-L) synthesis are increased, leading to osteoclast differentiation and activation a second time (24)(10)(14)(26) $(27)(28)(29)(30)$. Serum RANK-L is reported to be increased in ACPA-positive patients independent of acute phase reactants and pro-inflammatory cytokines, thus affirming the role of autoimmunity passing through ACPA itself on local osteoclastogenesis $^{(22)}$. Other mechanisms via OPG/Wnt/DKK1/sclerostin pathways have also been described ${ }^{(16)}$.

Both erosion and osteoporosis in RA are related to osteoclast activation via RANK-L pathway stimulation. However, the role of autoimmunity, especially ACPA, in the association between erosion and bone loss has only sparsely been studied $^{(31)}$. The primary objective of our study was to evaluate whether an association between erosion and bone mineral density in RA can be driven by ACPA status or titer. The secondary objectives were to evaluate whether other autoimmunity parameters, such as rheumatoid factor (RF) and anti-nuclear antibody (ANA), might have a role in this association.

\section{Patients And Methods Study population}

Patients followed for rheumatoid arthritis (RA) in our department from January 2008 to May 2019 were selected for this monocentric cross-sectional study based on clinical, biological and imaging data performed in daily practice. The diagnosis of RA was defined by 1987 ACR criteria or 2010 ACR-EULAR criteria for the most recent patients. To be included, patients had to undergo hand/foot radiography and biology at intervals of less than 2 years from DXA. Then, only patients with continuous quantitative values of ACPA titers were included. Figure 1 presents the flow chart of the inclusion and exclusion criteria.

\section{Clinical data}

Data were collected from the computerized medical records of our department: sex, age, BMI, tobacco use, disease duration, prior treatments (anti-osteoporotic, corticosteroids, DMARDs) and disease activity based on DAS28CRP.

\section{Biological and immunological data}

Levels of C-reactive protein (CRP) and erythrocyte sedimentation rate (ESR) were collected. The presence of inflammation was defined as a CRP value $>5 \mathrm{mg} / \mathrm{l}$.

Immunological status and antibody titers (ACPA, RF, ANA) were extracted from the internal laboratory information system database of the Department of Immunology. From 2008 to June 2011, anti-CCP2 dosage kits (Werfen, France) were used in our centre with a positive threshold for ACPA titer $\geq 25 \mathrm{IU} / \mathrm{L}$. ACPA titers are presented as continuous quantitative values. From July 2011, anti-CCP3 dosage kits (Werfen, France) were introduced. The threshold was also $25 \mathrm{IU} / \mathrm{L}$, but continuous values were only available until $250 \mathrm{IU} / \mathrm{L}$, and higher titers were $>250 \mathrm{IU} / \mathrm{L}$. As ACPA titers are correlated with inflammation and erosions, we decided to exclude patients without a continuous titer of anti-CCP, which means that all patients with a titer expressed as ">250 IU/L". For RF dosage, only IgM serotypes were considered, and titers were given in quantitative values with a positivity threshold of $20 \mathrm{IU} / \mathrm{L}$. ANA titer threshold was set as $\geq 16 \mathrm{IU} / \mathrm{L}$. 


\section{Dual-energy X-ray absorptiometry (DXA) assessment}

Dual-energy X-ray absorptiometry (DXA) data (Advance PA +301010, enCORE, version 14.10.022, Madison, WI, 53718, USA) were extracted from reports registered in our computerized database. We selected the T-score, Z-score and bone mineral density in $\mathrm{g} / \mathrm{cm}^{2}$ at the lumbar spine (L2-L4) and hip.

The presence of osteoporosis on DXA was defined by a T-score $\leq-2.5$, and the values were also expressed as continuous variables. We took T-score (as a quantitative value) and BMD at the hip as the primary endpoints, as they are criteria for the FRAX® score.

\section{Erosion assessment}

The assessment of erosions was performed by one reader (ICV) according to the modified Sharp/van der Heijde erosion score (SHSe) on radiographic postero-anterior views of hands and antero-posterior views of feet. Readings were performed blinded to the patient's clinical and biological information. An erosion with a grade $\geq 2$ was considered significant, and RA was considered erosive according to the EULAR 2013 definition ${ }^{(32)}$, i.e., presence of erosion on at least three separate joints among the following sites: proximal interphalangeal joints (PIP), metacarpophalangeal joints (MCP), wrist (counted as one joint), and metatarsophalangeal joints (MTP) on radiographs of both hands and feet ${ }^{(33)}$.

The total SHSe score and their subscores for hand and feet were calculated. We considered the SHSe score and erosive status to be primary endpoints.

\section{Statistical analysis}

The distribution of the variables was assessed by the Shapiro-Wilk normality test. Among the variables, only age, DAS28CRP and T-score followed a normal distribution.

Qualitative variables are shown as percentage. Quantitative variables are shown as medians (first and third quartiles) in cases of abnormal distribution and as the mean and standard deviation for normally distributed variables. In univariate analysis, for normally distributed variables, we used Student's t-test for quantitative variables and the Mann-Whitney $U$ test for qualitative variables. Among the other variables, we tested the association between two quantitative variables by using linear regression. For qualitative variables, the Fisher test was used. Only variables with a $p$ value $\leq 0,1$ were included in the multivariate analysis. A linear regression was performed to test variables significantly associated with the outcome in univariate analysis. The results were assessed at the $95 \%$ confidence interval, and a p value $<0.05$ was regarded as statistically significant. All statistical analyses were performed using XLSTAT version 2020.3.13 ®.

According to ethical considerations, no consent was needed since the data were extracted from regular medical records and anonymized for analysis. This study was recorded in the clinical research department according to the following number: 2020PI083.

\section{Results}

\section{Patients characteristics}

One hundred forty-nine patients fulfilled the inclusion criteria. Patients characteristics are presented in Table 1. There was a majority of women (75.8\%), and the patients' mean age was 62 (SD 9.61) years old. Additionally, there was a relatively long duration of RA, with a median disease duration of $132[60 ; 240]$ months. Most of them had moderate disease activity with a mean of 4.64 (SD 1.3) for DAS28CRP, and 79.8\% had current or past corticosteroid therapy. 
Smoking was recorded in $20.1 \%$ of the patients. In total, at least $83,9 \%$ of the patients had at least one risk factor for osteoporosis added to RA.

The mean T-scores were - 1.12 (SD 1.25) and - 0.78 (SD 1.54) at the hip and spine, respectively. Sixteen patients (10.7\%) had osteoporosis (i.e., T-score $\leq-2.5$ ) at the hip, and sixteen patients (10.7\%) had osteoporosis at the spine. Twenty-six (17.5\%) patients were classified as osteoporotic at least on site. Past or current anti-osteoporotic treatment was administered in $31.5 \%$ of the patients. A large proportion of patients $(79.9 \%)$ had an erosive disease. The median SHSe total score was 40.0 [15; 81]. ACPA and RF were positive in $61.1 \%$ and $57 \%$ of the RA patients, respectively.

ACPA-positive RA had a significantly higher disease activity index than ACPA-negative RA (DAS28CRP of 4.8 (SD 1.3) vs. 4.3 (SD 1.2); $p=0.02$ ). They were also more numerous having RF or ANA with significantly higher titers than ACPAnegative patients. The total SHSe score was higher in ACPA-positive RA patients, with a median of 49.5 [20.7; 93.5$]$ versus $29[12 ; 65](p=0.04)$ in ACPA-negative patients. Details are presented in Table 1. 
Table 1

Patient's characteristics

\begin{tabular}{|c|c|c|c|c|}
\hline Variables & $\begin{array}{l}\text { TOTAL }(n= \\
149)\end{array}$ & $\mathrm{ACPA}+(n=91)$ & $\begin{array}{l}\text { ACPA - }(n= \\
58)\end{array}$ & $\mathrm{p}$ value \\
\hline Demographic and Clinical Characteristics & $62.1(9.6)$ & $61.5(9.6)$ & $63.1(9.6)$ & 0.290 \\
\hline Age (years) & $113(75.8 \%)$ & $70(76.9 \%)$ & $43(74.1 \%)$ & 0.690 \\
\hline Women & $132[60 ; 240]$ & $156[84 ; 240]$ & $114[36 ; 234]$ & 0.060 \\
\hline Disease duration (months) & $4.64(1.3)$ & $4.8(1.3)$ & $4.3(1.2)$ & 0.023 \\
\hline DAS 28CRP & $26.1[0 ; 31.6]$ & $25.6[22.1 ; 27.9]$ & $26.5[23.5 ;$ & \\
\hline $\begin{array}{l}\text { BMI } \\
\text { Tobacco (currently) }\end{array}$ & $30(20.1 \%)$ & $16(17.6 \%)$ & $14(24.1 \%)$ & 0.390 \\
\hline Treatments & $118(79.2 \%)$ & $71(78.0 \%)$ & $47(81.0 \%)$ & 0.650 \\
\hline Corticosteroids: prior or current intake & $7.5[0 ; 10]$ & $7.5[0 ; 10]$ & $5[4.5 ; 10]$ & 0.380 \\
\hline Corticosteroid dose (mg/d) & $91(61.1 \%)$ & $57(62.6 \%)$ & $32(55.2 \%)$ & 0.350 \\
\hline $\begin{array}{l}\text { DMARDs: prior or current intake } \\
\text { Anti-osteoporotic treatment: prior or current } \\
\text { intake }\end{array}$ & $47(31.5 \%)$ & $25(27.5 \%)$ & $22(37.9 \%)$ & 0.120 \\
\hline Biology & $85(57.0 \%)$ & $67(73.6 \%)$ & $18(31.0 \%)$ & $<$ \\
\hline RF positive & $31.8[0 ; 130.5]$ & $\begin{array}{l}81.8[16.7 ; \\
198.2]\end{array}$ & & \\
\hline RF titer (IU/mL) & $122(81.9 \%)$ & $82(90.1 \%)$ & $40(69.0 \%)$ & 0.0001 \\
\hline ANA positive & $256.0[40 ; 512]$ & 256 [128; 1024$]$ & $128[0 ; 256]$ & $\hat{0} .0001$ \\
\hline ANA titer & $67(45.0 \%)$ & $103[34 \cdot 221]$ & $9.6[4.3 ; 17.2]$ & $0 \cap 01$ \\
\hline ACPA and RF positive & $9.9[3.4 ; 20]$ & $315[157 \cdot 492]$ & $27[20 ; 38]$ & 0580 \\
\hline $\begin{array}{l}\mathrm{CRP}(\mathrm{mg} / \mathrm{L}) \\
\mathrm{ESR} \text { (mm at } 1 \mathrm{st} \text { hour) }\end{array}$ & $29[17 ; 44]$ & & & 0.530 \\
\hline
\end{tabular}




\begin{tabular}{|c|c|c|c|c|}
\hline Variables & $\begin{array}{l}\text { TOTAL }(n= \\
\text { 149) }\end{array}$ & $\mathrm{ACPA}+(n=91)$ & $\begin{array}{l}\text { ACPA - }(n= \\
58)\end{array}$ & $\mathrm{p}$ value \\
\hline Imaging & $119(79.9 \%)$ & $76(83.5 \%)$ & $43(74.1 \%)$ & 0.390 \\
\hline Radiography & $14[5 ; 27]$ & $16.5[7 ; 30]$ & $12[3 ; 23]$ & 0.057 \\
\hline Erosive RA & $40.0[15 ; 81]$ & $49.5[20.7 ; 93.5]$ & $29[12 ; 65]$ & 0.040 \\
\hline $\mathrm{Nb}$ of $\geq$ grade 2 erosion & $21.0[5 ; 47]$ & $27[5.7 ; 55.2]$ & $18[3 ; 38]$ & 0.200 \\
\hline SHSe total score & $18.0[4 ; 36]$ & $20.5[7.7 ; 40.5]$ & $11.5[2 ; 28]$ & 0.010 \\
\hline SHSe hand score & $-1.1(1,3)$ & $-1.2(1.2)$ & $-1.0(1.3)$ & 0.502 \\
\hline SHSe foot score & $16(10.7 \%)$ & $9(9.9 \%)$ & $7(12.1 \%)$ & 0.900 \\
\hline DEXA & $-0.1(1.1)$ & $-0.2(1.0)$ & $0.1(1.2)$ & 0.200 \\
\hline T-score at hip & $0.9[0.8 ; 1]$ & $0.8[0.8 ; 0.9]$ & $0.9[0.8 ; 1]$ & 0.410 \\
\hline T-score $<-2.5$ at hip & $-0.8(1.5)$ & $-0.8(1.5)$ & $-0.8(1.6)$ & 0.909 \\
\hline Z-score at hip & $16(10.7 \%)$ & $8(8.8 \%)$ & $8.0(13.8 \%)$ & 0.310 \\
\hline BMD (g/cm2) at hip & $0.3(1.6)$ & $0.3(1.6)$ & $0.4(1.4)$ & 0.570 \\
\hline T-score at lumbar spine & $1.1[0.9 ; 1.2]$ & $1.1[0.9 ; 1.2]$ & $1.1[0.9 ; 1.2]$ & 0.974 \\
\hline T-score $<-2.5$ at lumbar spine & $26(17.5 \%)$ & $8(8.8 \%)$ & $9(15.5 \%)$ & 0.780 \\
\hline \multicolumn{5}{|l|}{ Z-score at lumbar spine } \\
\hline \multicolumn{5}{|l|}{ BMD $(\mathrm{g} / \mathrm{cm} 2)$ at lumbar spine } \\
\hline T-score $<-2.5$ at lumbar spine and/or hip & & & & \\
\hline
\end{tabular}

SD: standard deviation, Q1: first quartile, Q3: third quartile, sDMARD: synthetic disease modifying anti-rheumatic drug, ACPA: anti-cyclic citrullinated peptide antibodies, RF: rheumatoid factors, ANA: anti-nuclear antibodies, CRP: C-reactive protein, SHSe: modified Sharp/Van der Heijde erosion score, Nb: number, BMD: bone mineral density, d: day, NS: non*: significant

\section{Erosion assessment}

In univariate analysis, ACPA-positive patients had a significantly higher SHSe total score, with a mean score of 63.08 (SD 53.25) versus 45.55 (SD 44.06) in ACPA-negative RA ( $p=0.04$ ) (Table 2). Subanalyses of hand and feet SHSe scores showed similar results (data not shown). No association was demonstrated between erosive status and ACPA status or titers ( $p$ value $=0.12$ ). Significantly higher SHSe total scores were observed in RF-positive patients than in RFnegative patients: mean of 65.4 (SD 54.0) vs. 43.6 (SD 42.7), respectively ( $p=0.016)$. Double positivity (ACPA positive and RF positive) was significantly associated with erosive RA, with a prevalence of $90.5 \% v s .74 .7 \%$ ( $p$ value $=0.044)$. Nevertheless, titers of autoantibodies had no significant relation with total SHSe.

SHSe total score and SHSe subscores were inversely associated with BMD and T-score at hip, with the hip T-score determining approximately $16 \%$ of the SHSe total score $\left(R^{2}=0.158\right)($ Table 2$)$. Similar trends were observed for BMD and T-score at the spine (Table 2). The status of erosive disease was significantly associated with a lower hip BMD (0.831, SD 0.22 vs. 0.965 SD $0.16 ; p<0.0001)$. The status of erosive disease was also significantly associated with a 
lower hip T score of -1.33 (SD 1.1) vs. -0.35 (SD 1.3) $(\mathrm{p}<0.0001)$ for non-erosive RA. Similar trends were also observed in spine BMD and T-score.

Disease duration explained $31.5 \%$ of the SHSe total score variation $\left(R^{2}=0.315\right)$. Smokers had a significantly lower total SHSe than nonsmokers: 38.4 (SD 47.7) vs. 56.8 (SD 49.2), respectively ( $p=0.031)$. BMI was inversely associated with SHSe total score; for each increase of 1 unit for BMI, we had a decrease of $-0,243$ for the SHSe total score (value $0.243 ; p=0.019)$.

In multivariate analysis (Fig. 2), disease duration and hip BMD were independent factors for erosion severity evaluated on SHSe total score. When hip T-score was included in the linear regression model instead of hip BMD, the variables associated with the SHSe total score were disease duration and hip T-score. When the disease duration was excluded from the model, osteoporosis parameters (hip BMD and T-score) were still associated with the SHSe total score. 
Table 2

- Variables associated with erosions in univariate analysis

SHSe total score Erosive status

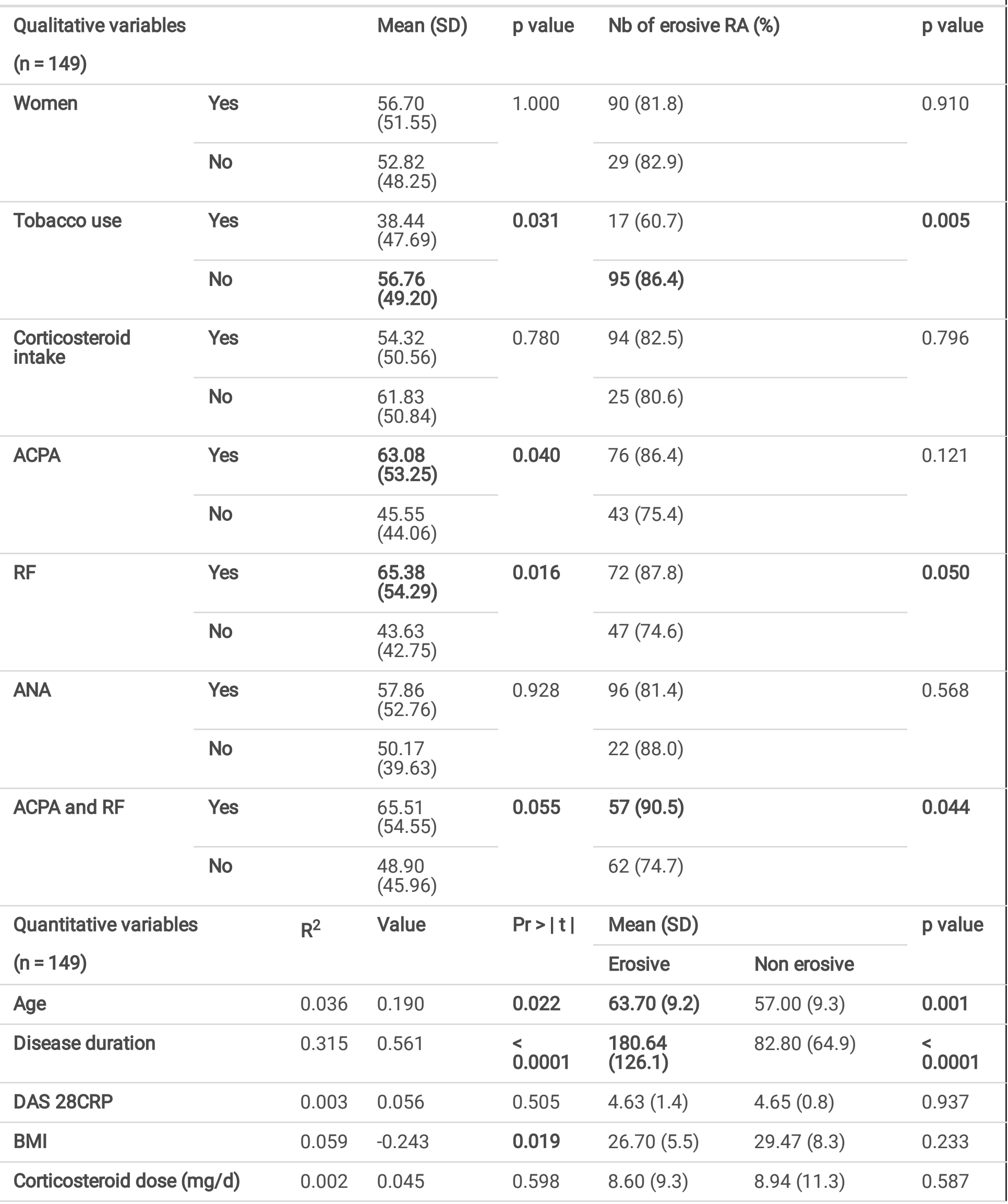




\begin{tabular}{|c|c|c|c|c|c|c|}
\hline \multirow[b]{2}{*}{ CRP } & \multicolumn{3}{|c|}{ SHSe total score } & \multicolumn{3}{|l|}{ Erosive status } \\
\hline & 0.001 & 0.024 & 0.779 & $19.50(26.2)$ & 13.85 (18.6) & 0.557 \\
\hline ACPA titer & 0.001 & 0.032 & 0.705 & $\begin{array}{l}480.80 \\
(760.4)\end{array}$ & $431.20(875)$ & 0.129 \\
\hline RF titer & 0.000 & 0.011 & 0.893 & 120.65 (179) & $108.80(278.5)$ & 0.090 \\
\hline ANA titer & 0.007 & 0.086 & 0.309 & $\begin{array}{l}929.30 \\
(3175)\end{array}$ & $\begin{array}{l}1960.70 \\
(6601.6)\end{array}$ & 0.830 \\
\hline Hip BMD & 0.049 & -0.222 & 0.019 & $0.83(0.2)$ & $0.97(0.2)$ & $\begin{array}{l}<.0001 \\
0 .\end{array}$ \\
\hline Hip T-score & 0.158 & -0.397 & $<0.001$ & $-1.33(1.1)$ & $-0.35(1.3)$ & 0.001 \\
\hline Spine BMD & 0.032 & -0.178 & 0.033 & $1.16(0.2)$ & $1.05(0.2)$ & 0.038 \\
\hline Spine T-score & 0.032 & -0.178 & 0.033 & $-0.95(1.4)$ & $-0.08(1.4)$ & 0.010 \\
\hline
\end{tabular}

SD: standard deviation, ACPA: anti-cyclic citrullinated peptide antibodies, RF: rheumatoid factors, ANA: anti-nuclear antibodies, CRP: C-reactive protein,

BMI: body mass index, SHSe: modified Sharp/Van der Heijde score, BMD: bone mineral density, Nb: number, d: day

Student's t-test, Mann-Whitney U-test, Khi2 or Fisher's test and linear regression were used.

\section{A: SHSe total score and associated variables with hip BMD included in the model}

\section{B: SHSe total score and associated variables with hip T-score included in the model.}

SHSe: modified Sharp/Van der Heijde erosion score, BMI: body mass index, BMD: bone mineral density, ACPA: anticyclic citrullinated peptide antibodies, RF: rheumatoid factors,

\section{Bone mineral density and T-score assessment}

The presence of ACPA was not significantly associated with hip or spine BMD (Tables 3 and 4, respectively). However, higher titers of ACPA were associated with lower BMD at the hip (value $=-0.216 ; p=0.01$ ) but not at the spine. Analysis of the Z-score did not provide supplementary information (data not shown). The presence of RF or ANA did not highlight any significant relation with either hip or lumbar spine BMD or T-score.

For the other demographic and clinical variables, women had a significantly lower BMD and T-score at the two sites and were significantly more often classified as osteoporotic at the hip ( $p$ value $=0.013$ ) (Table 3 ). BMI was positively associated with DXA parameters at both sites, i.e., patients with a higher BMI had higher BMD or T-score. Intake or dose of corticosteroids were not associated with lower values of BMD and T-score at hip and spine. Disease duration had a significant impact on BMD and T-score at hip and explained $12,6 \%$ of the variation of the hip T-score $\left(R^{2}=0.126\right)$. These associations were also observed for spine BMD (Table 4). Osteoporosis at the hip was associated with higher disease activity scores, with a mean DAS28CRP of 5.5 (SD 1.6) versus 4.6 (SD 1.2) in patients without osteoporosis $(p=0.007)$ (Table 3). The same results were observed for spine osteoporosis (Table 4).

As previously shown, hip BMD and T-score were inversely associated with SHSe total score. RA patients with erosive disease also had lower hip BMD and hip T-scores (Table 2 and Table 3). Similar results were observed for spine BMD and T-score (Table 4). 
In the linear regression model with hip BMD as the primary outcome, BMI, sex and SHSe total score were still independently associated with hip BMD. When erosive status was included in the model instead of SHSe total score, hip BMD was still independently associated with BMI, sex and erosive disease (Fig. 3A). When hip T-score was the primary outcome of the linear regression model, BMI, gender and SHSe total score were independently associated with hip Tscore. When erosive status was included instead of SHSe total score, hip T-score was still independently associated with BMI, sex and erosive disease (Fig. 3B) 


\begin{tabular}{|c|c|c|c|c|c|c|c|c|c|}
\hline & & HIP & & & & & & & \\
\hline Qualitative var & & & BMD $(g$ & cm2) & & T-score & & T-score (binary) & \\
\hline & & & $\begin{array}{l}\text { Mean } \\
\text { (SD) }\end{array}$ & $\begin{array}{l}\mathrm{p} \\
\text { value }\end{array}$ & & $\begin{array}{l}\text { Mean } \\
\text { (SD) }\end{array}$ & $\begin{array}{l}\mathrm{p} \\
\text { value }\end{array}$ & OR [Cl 95\%] & \\
\hline Women & Yes & & $\begin{array}{l}0.849 \\
(0.16)\end{array}$ & 0.003 & & $\begin{array}{l}-1.271 \\
(1.27)\end{array}$ & 0.018 & $0.185[0.033-1.031]$ & \\
\hline & No & & $\begin{array}{l}0.890 \\
(0.36)\end{array}$ & & & $\begin{array}{l}-0.688 \\
(1.06)\end{array}$ & & & \\
\hline Tobacco use & Yes & & $\begin{array}{l}0.877 \\
(0.17)\end{array}$ & 0.978 & & $\begin{array}{l}-1.125 \\
(1.30)\end{array}$ & 0.999 & $1.091[0.305-3.896]$ & \\
\hline & No & & $\begin{array}{l}0.855 \\
(0.24)\end{array}$ & & & $\begin{array}{l}-1.125 \\
(1.25)\end{array}$ & & & \\
\hline Corticosteroid & Yes & & $\begin{array}{l}0.852 \\
(0.23)\end{array}$ & 0.406 & & $\begin{array}{l}-1.172 \\
(1.21)\end{array}$ & 0.341 & $0.794[0.250-2.525]$ & \\
\hline & No & & $\begin{array}{l}0.894 \\
(0.17)\end{array}$ & & & $\begin{array}{l}-0.918 \\
(1.41)\end{array}$ & & & \\
\hline $\begin{array}{l}\text { Erosive } \\
\text { status }\end{array}$ & Yes & & $\begin{array}{l}0.835 \\
(0.23)\end{array}$ & 0.001 & & $\begin{array}{l}-1.311 \\
(1.18)\end{array}$ & 0.001 & $3.75[0.664-21.182]$ & \\
\hline & No & & $\begin{array}{l}0.971 \\
(0.16)\end{array}$ & & & $\begin{array}{l}-0.404 \\
(1.22)\end{array}$ & & & \\
\hline ACPA & Yes & & $\begin{array}{l}0.842 \\
(0.25)\end{array}$ & 0.400 & & $\begin{array}{l}-1.198 \\
(1.20)\end{array}$ & 0.492 & $0.814[0.293-2.257]$ & \\
\hline & No & & $\begin{array}{l}0.883 \\
(0.16)\end{array}$ & & & $\begin{array}{l}-1.048 \\
(1.29)\end{array}$ & & & \\
\hline RF & Yes & & $\begin{array}{l}0.831 \\
(0.25)\end{array}$ & 0.156 & & $\begin{array}{l}-1.278 \\
(1.12)\end{array}$ & 0.141 & $0.986[0.356-2.730]$ & \\
\hline & No & & $\begin{array}{l}0.893 \\
(0.17)\end{array}$ & & & $\begin{array}{l}-0.968 \\
(1.35)\end{array}$ & & & \\
\hline ANA & Yes & & $\begin{array}{l}0.843 \\
(0.23)\end{array}$ & 0.192 & & $\begin{array}{l}-1.229 \\
(1.24)\end{array}$ & 0.080 & $3.22[0.569-18.267]$ & \\
\hline & No & & $\begin{array}{l}0.916 \\
(0.14)\end{array}$ & & & $\begin{array}{l}-0.727 \\
(1.18)\end{array}$ & & & \\
\hline ACPA and RF & Yes & & $\begin{array}{l}0.823 \\
(0.27)\end{array}$ & 0.193 & & $\begin{array}{l}-1.281 \\
(1.09)\end{array}$ & 0.214 & $0.734[0.260-2.073]$ & \\
\hline & No & & $\begin{array}{l}0.888 \\
(0.17)\end{array}$ & & & $\begin{array}{l}-1.018 \\
(1.33)\end{array}$ & & & \\
\hline Quantitative v & ables & BMD & (cm2) & & T-score & & & T-score (binary) & \\
\hline & & $\mathrm{R}^{2}$ & Value & $\mathrm{Pr}>\mid \mathrm{t}$ & $\mathrm{R}^{2}$ & Value & $\mathrm{Pr}>\mid \mathrm{t}$ & Mean (SD) & p \\
\hline & & & & & & & & $\begin{array}{ll}\text { T-sc } & \text { T-sc } \\
\leq-2.5 & >2.5\end{array}$ & \\
\hline
\end{tabular}




\begin{tabular}{|c|c|c|c|c|c|c|c|c|c|}
\hline & HIP & & & & & & & & \\
\hline Age & 0.002 & -0.041 & 0.632 & 0.039 & -0.197 & 0.020 & $\begin{array}{l}61.5 \\
(9.8)\end{array}$ & $\begin{array}{l}62.2 \\
(9.6)\end{array}$ & 0.780 \\
\hline Disease duration & 0.052 & -0.228 & 0.007 & 0.126 & -0.355 & $\hat{0} .0001$ & $\begin{array}{l}201.2 \\
(127.9)\end{array}$ & $\begin{array}{l}151.3 \\
(120.2)\end{array}$ & 0.225 \\
\hline DAS 28CRP & 0.013 & -0.114 & 0.183 & 0.005 & -0.072 & 0.405 & $5.5(1.5)$ & $4.6(1.2)$ & 0.007 \\
\hline BMI & 0.218 & 0.467 & $\hat{0} .0001$ & 0.220 & 0.469 & $\hat{0} .0001$ & $\begin{array}{l}73.3 \\
(46.8)\end{array}$ & $\begin{array}{l}51.1 \\
(47.8)\end{array}$ & 0.054 \\
\hline $\begin{array}{l}\text { Corticosteroid dose } \\
(\mathrm{mg} / \mathrm{d})\end{array}$ & 0.000 & 0.039 & 0.810 & 0.000 & 0.012 & 0.894 & $\begin{array}{l}8.1 \\
(11.0)\end{array}$ & $8.3(8.4)$ & 0.471 \\
\hline CRP & 0.002 & -0.047 & 0.585 & 0.017 & -0.131 & 0.126 & $\begin{array}{l}32.6 \\
(42.6)\end{array}$ & $\begin{array}{l}16.1 \\
(21.0)\end{array}$ & 0.085 \\
\hline ACPA titer & 0.047 & -0.216 & 0.010 & 0.000 & -0.009 & 0.914 & $\begin{array}{l}272.3 \\
(452.2)\end{array}$ & $\begin{array}{l}489.6 \\
(787.8)\end{array}$ & 0.536 \\
\hline RF titer & 0.021 & -0.146 & 0.084 & 0.017 & -0.132 & 0.121 & $\begin{array}{l}143.3 \\
(309.6)\end{array}$ & $\begin{array}{l}117.0 \\
(189.4)\end{array}$ & 0.527 \\
\hline ANA titer & 0.000 & -0.015 & 0.862 & 0.002 & -0.050 & 0.562 & $\begin{array}{l}3303.2 \\
(8227.8)\end{array}$ & $\begin{array}{l}822.9 \\
(3051.2)\end{array}$ & 0.148 \\
\hline SHSe total score & 0.049 & -0.222 & 0.009 & 0.158 & -0.397 & $\begin{array}{l}<.0001 \\
0.00\end{array}$ & $\begin{array}{l}79.4 \\
(51.5)\end{array}$ & $\begin{array}{l}50.6 \\
(47.9)\end{array}$ & 0.054 \\
\hline
\end{tabular}

Table 3 - Variables associated with bone evaluation and osteoporosis (T-score $\leq-2.5)$ at hip in univariate analysis

SD: standard deviation, T-sc: T-score, ACPA: anti-cyclic citrullinated peptide antibodies, RF: rheumatoid factors, ANA: anti-nuclear antibodies, BMD: bone mineral density, BMI: body mass index, SHSe: modified Sharp/Van der Heijde erosion score, $\mathrm{Nb}$ : number, $\mathrm{d}$ : day

Student's t-test, Mann-Whitney U-test, Khi2 or Fisher's test and linear regression were used 
Variables associated with bone evaluation and osteoporosis (T-score $\leq-2.5)$ at the lumbar spine in univariate analysis

\section{LUMBAR SPINE}

$\begin{array}{llll}\text { Qualitative variables } & \text { BMD }(\mathrm{g} / \mathrm{cm} 2) & \text { T-score } & \text { T-score (binary) } \\ (n=149) & & \end{array}$

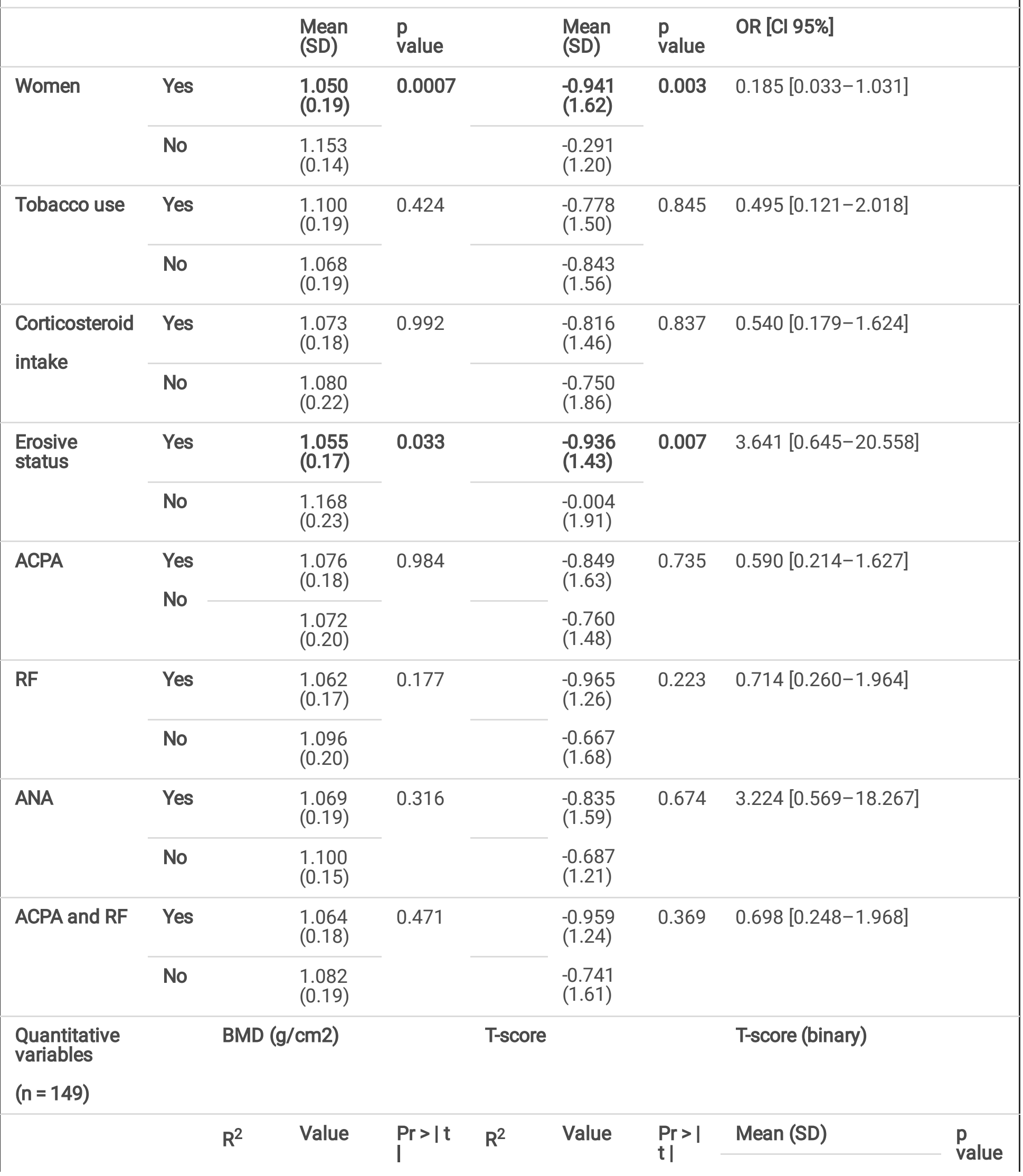




\begin{tabular}{|c|c|c|c|c|c|c|c|c|c|}
\hline \multicolumn{10}{|l|}{ LUMBAR SPINE } \\
\hline & & & & & & & $\begin{array}{l}\mathrm{T}-\mathrm{sc} \\
\leq-2.5\end{array}$ & $\begin{array}{l}\text { T-sc } \\
>-2.5\end{array}$ & \\
\hline Age & 0.001 & -0.028 & 0.734 & 0.001 & -0.029 & 0.730 & $61.7(9.8)$ & $\begin{array}{l}62.1 \\
(9.5)\end{array}$ & 0.872 \\
\hline Disease duration & 0.031 & -0.177 & 0.032 & 0.002 & 0.043 & 0.598 & $\begin{array}{l}73.3 \\
(46.8)\end{array}$ & $\begin{array}{l}160.9 \\
(127.9)\end{array}$ & 0.009 \\
\hline DAS 28CRP & 0.002 & -0.046 & 0.584 & 0.007 & 0.085 & 0.305 & $5.3(1.6)$ & $4.6(1.2)$ & 0.032 \\
\hline BMI & 0.061 & 0.247 & 0.018 & 0.058 & 0.242 & 0.021 & $26.0(4.2)$ & $\begin{array}{l}27.7 \\
(6.9)\end{array}$ & 0.671 \\
\hline $\begin{array}{l}\text { Corticosteroid dose } \\
(\mathrm{mg} / \mathrm{d})\end{array}$ & 0.000 & 0.011 & 0.899 & 0.000 & 0.000 & 0.997 & $6.7(10.3)$ & $9.1(9.6)$ & 0.069 \\
\hline CRP & 0.003 & -0.053 & 0.527 & 0.003 & -0.059 & 0.485 & $\begin{array}{l}23.1 \\
(32.9)\end{array}$ & $\begin{array}{l}17.7 \\
(23.8)\end{array}$ & 0.485 \\
\hline ACPA titer & 0.001 & 0.028 & 0.741 & 0.033 & -0.182 & 0.026 & $\begin{array}{l}308.0 \\
(409.1)\end{array}$ & $\begin{array}{l}502.1 \\
(816.8)\end{array}$ & 0.777 \\
\hline RF titer & 0.012 & -0.110 & 0.186 & 0.018 & -0.135 & 0.102 & $\begin{array}{l}128.1 \\
(177.9)\end{array}$ & $\begin{array}{l}116.5 \\
(206.0)\end{array}$ & 0.947 \\
\hline ANA titer & 0.014 & -0.118 & 0.158 & 0.001 & -0.024 & 0.769 & $\begin{array}{l}2888.3 \\
(8170.8)\end{array}$ & $\begin{array}{l}878.8 \\
(3069.5)\end{array}$ & 0.695 \\
\hline SHSe total score & 0.032 & -0.178 & 0.033 & 0.032 & -0.178 & 0.033 & $\begin{array}{l}52.5 \\
(43.6)\end{array}$ & $\begin{array}{l}56.6 \\
(51.5)\end{array}$ & 0.976 \\
\hline
\end{tabular}

SD: standard deviation, T-sc: T-score, ACPA: anti-cyclic citrullinated peptide antibodies, RF: rheumatoid factors, ANA: anti-nuclear antibodies, BMD: bone mineral density, BMI: body mass index, SHSe: modified Sharp/Van der Heijde erosion score, $\mathrm{Nb}$ : number, d: day

Student's t-test, Mann-Whitney U-test, Khi2 or Fisher's test and linear regression were used.

\section{A: Hip T-score and associated variables.}

\section{B: Hip BMD and associated variables}

SHSe: modified Sharp/Van der Heijde erosion score, BMI: body mass index, BMD: bone mineral density, ACPA: anticyclic citrullinated peptide antibodies

\section{Discussion}

Our aim was to determine for the first time whether bone fragility on the axial skeleton assessed by DXA (BMD and Tscore at hip) and erosion on peripheral joints evaluated by SHSe total score may be driven by ACPA and/or other autoimmunity-related antibodies (RF and ANA). We found that erosions (based on SHSe score) were associated with lower BMD and T-score at hip but also at spine. This association was confirmed at the patient level when erosive status (at least 3 erosive joints) was evaluated. Nevertheless, our study failed to demonstrate a role of ACPA (status or titers) in this association. 
The major factors for erosion (SHSe total score) were disease duration, hip T-score and BMD. This last association was also described by Bruno et al. in a cohort of early RA patients $(n=128)^{(14)}$. The exclusion of disease duration did not change the results, and hip T-score or BMD included separately were still significant independent factors for erosion severity. Univariate analysis showed that BMI and tobacco use were associated with lower erosion scores. Concerning BMI, Rydell et al. showed, in a population of 233 early RA ( $<12$ months of disease duration), that high BMI might reduce the risk of severe joint damage assessed also by the modified Sharp-Van der Heijde score ${ }^{(34)}$. In the Espoir cohort, Vesperini et al. showed that smokers had reduced radiographic progression at one year ${ }^{(35)}$. Variables associated with joint erosion on the modified Sharp-Van der Heijde erosion score in univariate analysis were the presence of ACPA, RF and double positivity. These relations are already known and previously reported in the literature ${ }^{(7)}$.

In our RA patients, $17.5 \%$ had osteoporosis at the hip and/or spine based on DXA assessment. This result is consistent with the literature; indeed, the described prevalence of densitometric osteoporosis ranges from 10 to $50 \%(14)(15)$. A large proportion of our patients presented at least one osteoporotic risk factor (84\%), and $31.5 \%$ were treated or had been treated with at least one anti-osteoporotic treatment. As previously reported, we showed that hip BMD or hip T-score were significantly associated with BMI and sex in multivariate analysis. Obviously, women had lower BMD and T-scores at the hip, and BMI is known to be protective against bone loss ${ }^{(14)}$.

We show that ACPA titers and not status are associated with lower BMD but were not found to be independent factors in multivariate analysis. In 578 early RA patients, Llorente et al. found that ACPA-positive RA patients were significantly associated with lower BMD at the hip and spine in both univariate and multivariate analyses ${ }^{(21)}$. Orsolini et al. evaluated only the Z score at both sites (spine and hip) in 127 RA patients. They failed to demonstrate any correlation between ACPA status and Z-score at the total femur, femoral neck or spine ${ }^{(36)}$, but some associations were observed according to the threshold of positivity when the analysis was performed on quartiles. In our study, analysis of Z-scores did not provide any supplementary information (data not shown). Interestingly, Orsolini et al. did not demonstrate any association between Z-score and RF (status or titer). In a Dutch cohort ( $n=408$ ) of early RA, Amkreutz et al. showed lower BMD at the spine and hip in ACPA-positive patients than in ACPA-negative patients at baseline, without the influence of ACPA levels and without significant changes over time. The difference in BMD did not reach the level of significance in the Swedish cohort $(n=198)$ at baseline in ACPA patients (status or titers), and no change over time was noted between the two subsets. Concerning RF, in the Dutch cohort, BMD was lower at the spine in RF-positive patients than in RF-negative patients at baseline in univariate analysis. This was not confirmed in multivariate analysis, and there was no significant variation over time ${ }^{(37)}$. Finally, Bruno et al. showed that osteopenia and osteoporotic status were associated with ACPA status in a population of early RA patients $(n=71)$ only in univariate analysis ${ }^{(14)}$. Autoimmunity seems to be less preponderant in long-duration RA. Indeed, in an established population of 149 RA patients - not early rheumatisms - we did not find any relation driven by ACPA. In summary, the role of autoimmunity seems to be modest, and the role of ACPAs at disease onset should be clarified in further studies. Controversial results may be related to the size of the sample and the proportion of patients with ACPA-positive status.

The strengths of our study include a satisfying proportion of ACPA-positive RA (61.8\%). Additionally, we evaluated a large proportion of patients with erosive status (almost $80 \%$ ) and a large range of SHSe total scores. The assessment of erosive status is particularly interesting in daily practice, as it is easy to determine if the patient has at least three eroded joints, whereas SHSe score analysis is poorly adapted in clinical practice, as it is much more complex and timeconsuming and rather suitable for clinical trials. Additionally, data for imaging (X-ray, DXA), biology (status and titers) and clinical variables were recorded within no longer than 2 years.

The limitations of our study are obviously the retrospective design with missing data. The fact that we excluded 129 patients without continuous titers of ACPAs reduced the sample size to 149 patients.

Page $16 / 22$ 
This possibly led to an insufficient number of patients to show a relation with ACPA in multivariate analysis.

Furthermore, studies on HRpQCT showed that the distal radius and tibia presented alterations in both cortical and trabecular bone parameters in RA patients compared to healthy individuals ${ }^{(30)(38)}$. Nevertheless, the impact of autoimmunity and osteoporosis on HRpQCT alterations has not yet been studied. In clinical practice, CT scans offer new approaches to evaluate trabecular bone density by the scanographic bone coefficient (SBAC-L1) of the first lumbar vertebra measurement and the trabecular bone architecture through the fractal dimension ${ }^{(11)(39)(40)(41)}$. Moreover, this imaging modality offers the opportunity to also diagnose vertebral fractures, which are a main complication of osteoporosis with higher morbidity risk ${ }^{(9)}$.

\section{Conclusion}

In summary, we have shown for the first time that the relationship between erosion and systemic bone loss in RA does not seem to be driven by ACPA or other autoimmunity-related antibodies but rather by disease duration. However, erosive RA should lead to osteoporotic screening to initiate as soon as possible anti-osteoporotic treatment in RA patients with bone fragility risks.

\section{Abbreviations}

SD

standard deviation

T-sc

T-score

ACPA

anti-cyclic citrullinated peptide antibodies

RF

rheumatoid factors

ANA

anti-nuclear antibodies

BMD

bone mineral density

BMI

body mass index

SHSe

modified Sharp/Van der Heijde erosion score

$\mathrm{Nb}$

number

d

day

\section{Declarations}

\section{Declarations}

\section{Ethics approval and consent to participate}


All procedures performed in this study were in accordance with the ethical considerations of the $1964 \mathrm{Helsinki}$ declaration and its later amendments or comparable ethical standards. This study was recorded in the clinical research department of Nancy according to the following number: 2020PI083.

\section{Consent for publication}

Not applicable.

\section{Declarations}

\section{Competing interests}

The authors declare that they have no competing interests

\section{Funding}

The authors declare that they did not receive any funding for this study.

\section{Author's contributions}

DL and MM conceived the study. MM and ICV collected data on erosions. MCB supervised the generation and collection of data for immunological parameters. MM, CM and DL collected other data. DL and MM performed the statistical analysis supervised by EA. All authors contributed to the article and approved the submitted version.

\section{ACKNOWLEDGEMENTS}

Not applicable.

\section{References}

1. Rudan I, Sidhu S, Papana A, Meng S, Xin-Wei Y, Wang W, et al. Prevalence of rheumatoid arthritis in low- and middle-income countries: A systematic review and analysis. J Glob Health June. 2015. doi:10.7189/jogh.05.010409.

2. Guillemin F, Saraux A, Guggenbuhl P, Roux C, Fardellone P, Le Bihan E, et al. Prevalence of rheumatoid arthritis in France: 2001. Ann Rheum Dis oct. 2005;64(10):1427-30.

3. Periarticular Bone Loss in Arthritis Is Induced by Autoantibodies Against. Citrullinated Vimentin - Engdahl - 2017 Journal of Bone and Mineral Research doi.org/10.1002/jbmr.3158.

4. Sokolove J, Pisetsky D. Bone loss, pain and inflammation: three faces of ACPA in RA pathogenesis. Ann Rheum DisApril. 2016;75(4):637-9.

5. Hauser B, Harre U. The Role of Autoantibodies in Bone Metabolism and Bone Loss. Calcif Tissue Int May. 2018;102(5):522-32.

6. Maddali Bongi S, Manetti R, Melchiorre D, Turchini S, Boccaccini P, Vanni L, et al. Anti-cyclic Citrullinated Peptide Antibodies are Highly Associated with Severe Bone Lesions in Rheumatoid Arthritis Anti-CCP and Bone Damage in RA. Autoimmunity September. 2004;37(6-7):495-501. 
7. Grosse J, Allado E, Roux C, Pierreisnard A, Couderc M, Clerc-Urmes I, et al. ACPA-positive versus ACPA-negative rheumatoid arthritis: two distinct erosive disease entities on radiography and ultrasonography. Rheumatol Int April. 2020;40(4):615-24.

8. Jilani AA, Mackworth-Young CG. The Role of Citrullinated Protein Antibodies in Predicting Erosive Disease in Rheumatoid Arthritis: A Systematic Literature Review and Meta-Analysis. Int J Rheumatol. 2015;2015:1-8.

9. Kweon S-M, Sohn DH, Park J-H, Koh JH, Park E-K, Lee H-N, et al. Male patients with rheumatoid arthritis have an increased risk of osteoporosis: Frequency and risk factors. Medicine (Baltimore). June 2018;97(24):e11122.

10. Adami G, Saag KG. Osteoporosis Pathophysiology, Epidemiology, and Screening in Rheumatoid Arthritis. Curr Rheumatol Rep July. 2019;21(7):34.

11. Hoes JN, Bultink IE, Lems WF. Management of osteoporosis in rheumatoid arthritis patients. Expert Opin Pharmacother March. 2015;16(4):559-71.

12. Okano T, Inui K, Tada M, Sugioka Y, Mamoto K, Wakitani S, et al. High frequency of vertebral fracture and low bone quality in patients with rheumatoid arthritis-Results from TOMORROW study. Mod Rheumatol May. 2017;27(3):398-404.

13. Jin S, Hsieh E, Peng L, Yu C, Wang Y, Wu C, et al. Incidence of fractures among patients with rheumatoid arthritis: a systematic review and meta-analysis. Osteoporos Int June. 2018;29(6):1263-75.

14. Bruno D, Fedele AL, Tolusso B, Barini A, Petricca L, Di Mario C, et al. Systemic Bone Density at Disease Onset Is Associated With Joint Erosion Progression in Early Naive to Treatment Rheumatoid Arthritis: A Prospective 12Month Follow-Up Open-Label Study. Front Med February. 2021. doi:10.3389/fmed.2021.613889.

15. Sinigaglia L, Varenna M, Girasole G, Bianchi G. Epidemiology of osteoporosis in rheumatic diseases. Rheum Dis Clin North Am November. 2006;32(4):631-58.

16. Llorente I, García-Castañeda N, Valero C, González-Álvaro I, Castañeda S. Osteoporosis in Rheumatoid Arthritis: Dangerous Liaisons. Front Med November. 2020;7:601618.

17. Mullen MB, Saag KG. Evaluating and mitigating fracture risk in established rheumatoid arthritis. Best Pract Res Clin Rheumatol August. 2015;29(4-5):614-27.

18. Joffe I, Epstein S. Osteoporosis associated with rheumatoid arthritis: Pathogenesis and management. Semin Arthritis RheumFebruary. 1991;20(4):256-72.

19. Cheng T-T, Lai H-M, Yu S-F, Chiu W-C, Hsu C-Y, Chen J-F, et al. The impact of low-dose glucocorticoids on disease activity, bone mineral density, fragility fractures, and 10-year probability of fractures in patients with rheumatoid arthritis. J Investig Med août. 2018;66(6):1004-7.

20. Tomizawa T, Ito H, Murata K, Hashimoto M, Tanaka M, Murakami K, et al. Distinct biomarkers for different bones in osteoporosis with rheumatoid arthritis. Arthritis Res Ther December. 2019;21(1):174.

21. Llorente I, Merino L, Ortiz AM, Escolano E, González-Ortega S, García-Vicuña R, et al. Anti-citrullinated protein antibodies are associated with decreased bone mineral density: baseline data from a register of early arthritis patients. Rheumatol Int May. 2017;37(5):799-806.

22. Bugatti S, Bogliolo L, Vitolo B, Manzo A, Montecucco C, Caporali R. Anti-citrullinated protein antibodies and high levels of rheumatoid factor are associated with systemic bone loss in patients with early untreated rheumatoid arthritis. Arthritis Res Ther December. 2016;18(1):226.

23. Cheng T-T, Yu S-F, Su F-M, Chen Y-C, Su BY-J, Chiu W-C, et al. Anti-CCP-positive patients with RA have a higher 10year probability of fracture evaluated by FRAX®: a registry study of RA with osteoporosis/fracture. Arthritis Res Ther December. 2018;20(1):16.

24. on behalf of the IOF Chronic Inflammation and Bone Structure (CIBS) Working Group. Zerbini CAF, Clark P, MendezSanchez L, Pereira RMR, Messina OD, et al. Biologic therapies and bone loss in rheumatoid arthritis. Osteoporos Int 
February 2017;28(2):429-46.

25. Kocijan R, Harre U, Schett G. ACPA and Bone Loss in Rheumatoid Arthritis. Curr Rheumatol Rep October. 2013;15(10):366.

26. Vis M, Güler-Yüksel M, Lems WF. Can bone loss in rheumatoid arthritis be prevented? Osteoporos Int October. 2013;24(10):2541-53.

27. Geusens P, Lems WF. Osteoimmunology and osteoporosis. Arthritis Res Ther. 2011;13(5):242.

28. Roux C. Osteoporosis in inflammatory joint diseases. Osteoporos Int févr. 2011;22(2):421-33.

29. Harre U, Georgess D, Bang H, Bozec A, Axmann R, Ossipova E, et al. Induction of osteoclastogenesis and bone loss by human autoantibodies against citrullinated vimentin. J Clin Invest May. 2012;122(5):1791-802.

30. Schett G. Autoimmunity as a trigger for structural bone damage in rheumatoid arthritis. Mod Rheumatol March. 2017;27(2):193-7.

31. Guler H, Turhanoglu AD, Ozer B, Ozer C, Balci A. The relationship between anti-cyclic citrullinated peptide and bone mineral density and radiographic damage in patients with rheumatoid arthritis. Scand $\mathrm{J}$ Rheumatol January. 2008;37(5):337-42.

32. Heijde D van der, Mil AHM, van der H, Aletaha, Bingham D, Burmester CO, Dougados GR. M, et al. EULAR definition of erosive disease in light of the 2010 ACR/EULAR rheumatoid arthritis classification criteria. Ann Rheum Dis April. 2013;72(4):479-81.

33. Roux C, Gandjbakhch F, Pierreisnard A, Couderc M, Lukas C, Masri R, et al. Ultrasonographic criteria for the diagnosis of erosive rheumatoid arthritis using osteoarthritic patients as controls compared to validated radiographic criteria. Joint Bone Spine July. 2019;86(4):467-74.

34. Rydell E, Forslind K, Nilsson J-Å, Jacobsson LTH, Turesson C. Smoking, body mass index, disease activity, and the risk of rapid radiographic progression in patients with early rheumatoid arthritis. Arthritis Res Ther. 2018;20:82.

35. Vesperini V, Lukas C, Fautrel B, Loet XL, Rincheval N, Combe B. Association of Tobacco Exposure and Reduction of Radiographic Progression in Early Rheumatoid Arthritis: Results From a French Multicenter Cohort. Arthritis Care Res. 2013;65(12):1899-906.

36. Orsolini G, Caimmi C, Viapiana O, Idolazzi L, Fracassi E, Gatti D, et al. Titer-Dependent Effect of Anti-Citrullinated Protein Antibodies On Systemic Bone Mass in Rheumatoid Arthritis Patients. Calcif Tissue Int July. 2017;101(1):17-23.

37. Amkreutz JAMP, de Moel EC, Theander L, Willim M, Heimans L, Nilsson J-Å, et al. Association Between Bone Mineral Density and Autoantibodies in Patients With Rheumatoid Arthritis. Arthritis Rheumatol Hoboken NJ June. 2021;73(6):921-30.

38. Jin S, Li M, Wang Q, Zeng X, Xia W, Yu W, et al. Bone mineral density and microarchitecture among Chinese patients with rheumatoid arthritis: a cross-sectional study with HRpQCT. Arthritis Res Ther April. 2021;23(1):127.

39. Fauny M, Albuisson E, Bauer E, Perrier-Cornet J, Chary-Valckenaere I, Loeuille D. Study of vertebral fracture and Scanographic Bone Attenuation Coefficient in rheumatoid arthritis and ankylosing spondylitis vs. controls. Sci Rep December. 2019;9(1):13323.

40. Perrier-Cornet J, Omorou AY, Fauny M, Loeuille D, Chary-Valckenaere I. Opportunistic screening for osteoporosis using thoraco-abdomino-pelvic CT-scan assessing the vertebral density in rheumatoid arthritis patients. Osteoporos Int June. 2019;30(6):1215-22.

41. Juhász B, Gulyás $K$, Horváth Á, Pethő $Z$, Bhattoa HP, Váncsa A, et al. Comparison of peripheral quantitative computed tomography forearm bone density versus DXA in rheumatoid arthritis patients and controls. Osteoporos Int April. 2017;28(4):1271-7. 


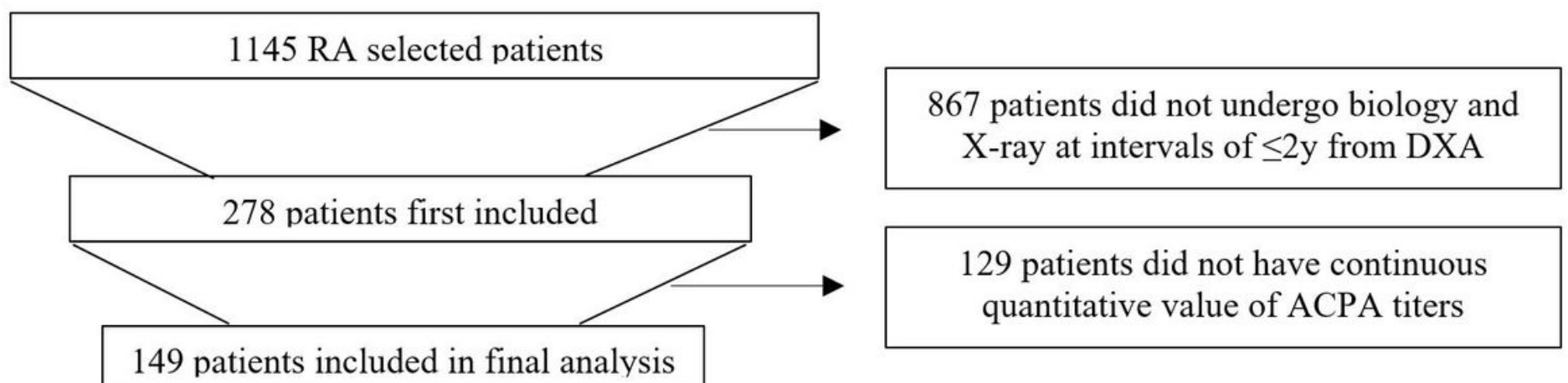

Figure 1

Study flow chart of the inclusion and exclusion criteria
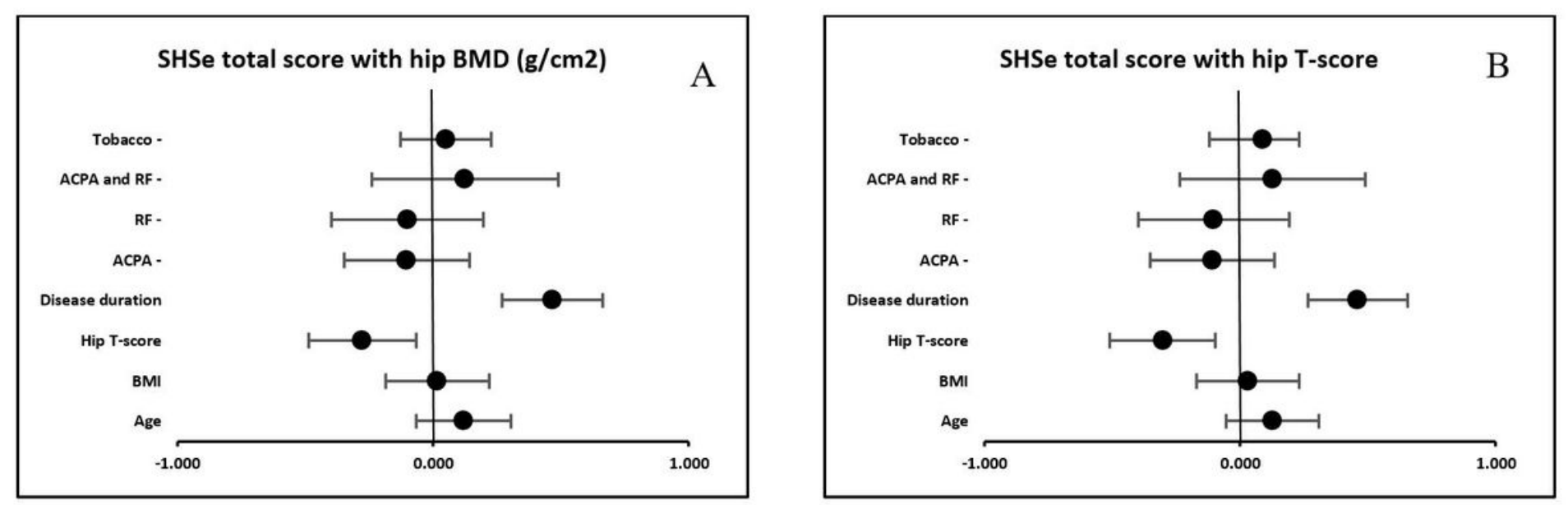

Figure 2

Multivariate analysis: a linear regression. A: SHSe total score and associated variables with hip BMD included in the model B: SHSe total score and associated variables with hip T-score included in the model. SHSe: modified Sharp/Van der Heijde erosion score, BMI: body mass index, BMD: bone mineral density, ACPA: anti-cyclic citrullinated peptide antibodies, RF: rheumatoid factors,
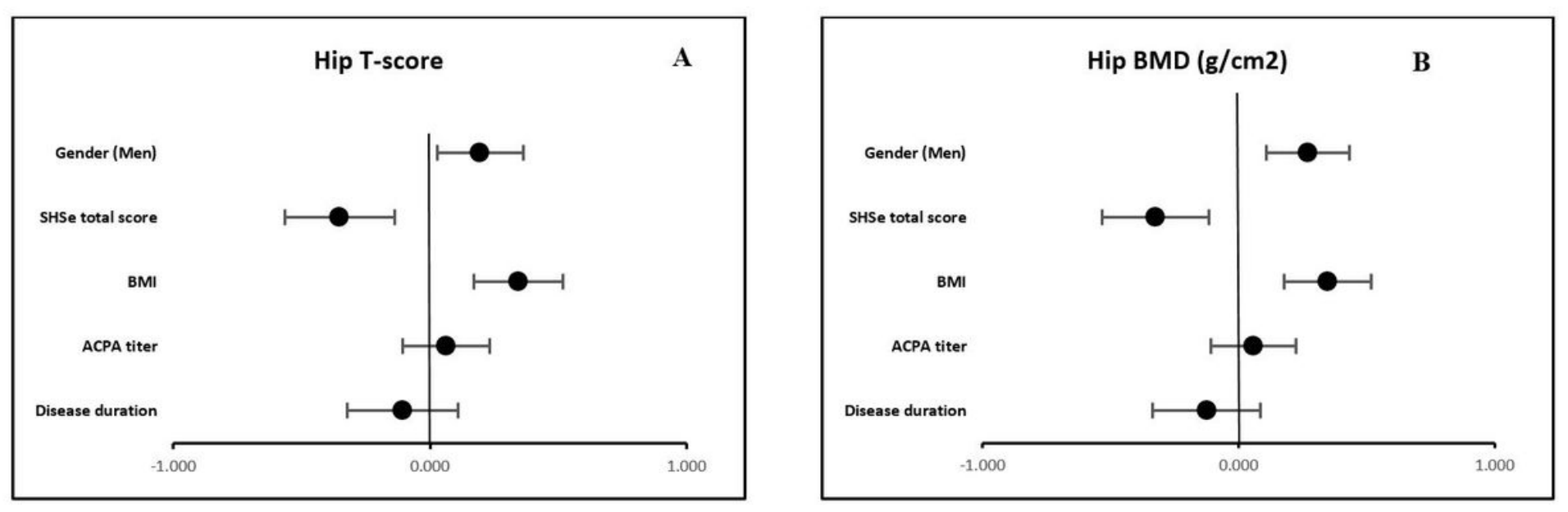


\section{Figure 3}

Multivariate analysis: a linear regression. A: Hip T-score and associated variables. B: Hip BMD and associated variables SHSe: modified Sharp/Van der Heijde erosion score, BMI: body mass index, BMD: bone mineral density, ACPA: anticyclic citrullinated peptide antibodies 